\title{
Probing flavor changing interactions in hadron collisions *
}

\author{
Chang Chao-Hsia ${ }^{a, b}$, Han Liang ${ }^{c}$, Jiang $\mathrm{Yi}^{c}$, Ma Wen-Gan ${ }^{a, b, c}$, Zhou Hong ${ }^{c}$, Zhou Mian-Lai ${ }^{c}$ \\ ${ }^{a}$ CCAST (World Laboratory), P.O.Box 8730, Beijing 100080, China. \\ ${ }^{b}$ Institute of Theoretical Physics, Academia Sinica, \\ P.O.Box 2735, Beijing 100080, China. \\ ${ }^{c}$ Department of Modern Physics, University of Science and Technology \\ of China (USTC), Hefei, Anhui 230027, China.
}

\begin{abstract}
The subprocess $g g \rightarrow t \bar{c}+\bar{t} c$ in the two-Higgs-doublet model with flavor-changing scalar couplings is examined at the one loop level. With perturbative QCD factorization theorem, the corresponding cross sections for hadron-hadron collisions are computed numerically. The results are applicable to the whole mass range of the weakly coupled Higgs bosons. In case we could efficiently exclude the severe backgrounds of the $t \bar{c}(\bar{t} c)$ production signal, probing the flavor-changing top-charmscalar vertex at hadron colliders would be very promising and accessible experimentally.

PACS number(s):13.85.Ni, 11.30.Hv, 12.60.Fr, 14.65.Ha
\end{abstract}

\footnotetext{
${ }^{*}$ This work was supported by National Natural Science Foundation of China.
} 


\section{Introduction}

There are stringent experimental constraints against the existence of tree level neutral flavor changing interactions, especially for light quarks[回]. This leads to the naturalness to suppress the flavor changing neutral current interactions (FCNCs) for all kinds of model building studies, that is realized in terms of the Glashow-Iliopoulos-Maiani (GIM) mechanism in the standard model(SM).

The two-Higgs-doublet models(THDMs) are extensions of the SM in which one more scalar doublet is added. In order to forbid possible tree level FCNCs to appear in the models, Glashow and Weinberg[3] proposed a neutral flavor conservation (NFC) condition by imposing discrete symmetries on the models. The common THDMs with the NFC condition can be divided into two categories, i.e., Model I and Model II. In Model I, both the up- and down-type quarks couple to the same one of the Higgs doublets, but in the Model II the up- and down-type quarks couple to the two Higgs doublets respectively. In fact, the suppression facts of the FCNC processes in the cases involving the light down-type quark sector have been observed experimentally [4, whereas those relevant to the up-type quark sector have not been established so well. Therefore the so-called Model-III of the THDM is proposed and it allows the FCNC Yukawa couplings which has the character that the couplings are related to the masses of the coupled flavors even at tree level[2].

As pointed out by Cheng, Sher and other authors [6] [7] [9], the Yukawa couplings are typically proportional to the masses of the coupled fermions at the vertices, it is rather natural to imagine having such Yukawa couplings for the FCNC interactions instead of placing the constraints on the theory. In this case, low energy limits on the FCNCs may be evaded, because the flavor changing couplings to the light quarks are small, and the suppression of the FCNC processes involving light quarks can be automatically satisfied. Then the imposition of discrete symmetries to obtain the NFC condition, therefore, is unnecessary, which is normally invoked in common THDMs to prevent the FCNC interactions at tree level. In the literature such the THDM without the NFC condition is called as the 'third' THDM (i.e., THDM III) [9], where the up-type and down-type quarks are allowed simultaneously to couple to more than one scalar doublet. In the framework of the model the effects of the FCNC interactions involving the heavy quark will be enhanced.

Now the top quark has been observed and a unexpected very large mass $m_{t}=173.8 \pm 5.2 \mathrm{GeV}$ (world average value) has been recognized[5]. This 'extraordinary' mass scale of the top quark may have many important implications pertaining to many outstanding issues. One of them is to test the FCNC processes requested in the Model-III. Namely, it is a 'good place' to observe the facts of the Model-III for the FCNC interactions. The measurement of FCNC processes involving top quark would provide an important test for the discrimination of various models. In the THDM III one would expect that large effects of the FCNC interactions could manifest themselves in the cases involving the massive top quark. Therefore testing the existence of the flavor changing scalar interactions involving top quarks are clearly important. D. Atwood et al. [10] presented results of a calculation for the process $e^{+} e^{-} \rightarrow t \bar{c}\left(\right.$ or $\bar{t} c$ )in the THDM III, and they obtained $R^{t c} / \lambda^{4}$ to be in the order of $10^{-5}$ with proper parameters. Recently, Jiang et al. 11] studied the production rates of $e^{+} e^{-} \rightarrow \gamma \gamma \rightarrow t \bar{c}+\bar{t} c$ for the NLC and found that this process is more promising than the straight production via $e^{+} e^{-}$collisions for probing the FCNC interactions. Abraham et al. 12. also 
investigated the anomalous $\bar{t} q \gamma$ couplings via single top quark production processes by considering effective Lagrangian of the lowest dimension with $\gamma \gamma$ collisions, and found the processes would be observable with suitable strength of anomalous coupling as long as b-tagging and suitable kinematic cuts are taken properly.

After the termination of the running of the LEP2, the hadron colliders Tevatron and LHC will be the only machines in searching for the FCNC processes. It is believed that more experimental events involving top quark will be collected in these hadronic machines. It will give a good chance to study the physics relevant to the FCNC processes of the Model-III. In this paper we are to study the problem and present complete one-loop calculation for the subprocess $g g \rightarrow t \bar{c}$ (or $\bar{t} c$ ) to the order $O\left(m_{t} m_{c} / m_{W}^{2}\right)$ for the THDM Model-III. In fact, the obtained results in the paper are applicable to the whole mass range for weakly coupled Higgs bosons. The production cross sections of $p p(\bar{p}) \rightarrow g g \rightarrow t \bar{c}+\bar{t} c+X$ are also given for the Tevatron and LHC. The paper is organized as follows: The details of the calculation are given in Sec. II. In Sec. III numerical results, discussions and a short summary are presented. Finally, the explicit expressions used in the paper are collected in appendix.

\section{Calculation}

In the third type of the two-Higgs-doublet model, the up-type and down-type quarks are allowed simultaneously to couple to more than one scalar doublet. Since there is no global symmetry that distinguishes the two doublets in the model, we will assume that only one of them $\left(\phi_{1}\right)$ develops a vacuum expectation value and the second one $\left(\phi_{2}\right)$ remains unbroken, i.e.

$$
<\phi_{1}>=\left(\begin{array}{l}
0 \\
v / \sqrt{2}
\end{array}\right), \quad<\phi_{2}>=0
$$

where $v \simeq 246 \mathrm{GeV}$. The physical spectrum of Higgs bosons consists of two scalar neutral bosons $h^{0}$ and $H^{0}$, one pseudoscalar neutral boson $A^{0}$ and two charged Higgs $H^{ \pm}$,

$$
\begin{aligned}
& H^{0}=\sqrt{2}\left[\left(\operatorname{Re} \phi_{1}^{0}-v\right) \cos \alpha+\operatorname{Re} \phi_{2}^{0} \sin \alpha\right], \\
& h^{0}=\sqrt{2}\left[-\left(\operatorname{Re} \phi_{1}^{0}-v\right) \sin \alpha+\operatorname{Re} \phi_{2}^{0} \cos \alpha\right], \\
& A^{0}=\sqrt{2}\left(-\operatorname{Im} \phi_{2}^{0}\right) .
\end{aligned}
$$

The masses of the five neutral and charged Higgs bosons and the mixing angle $\alpha$ are free parameters of the model. The Yukawa couplings to quarks are[2],

$$
\mathcal{L}_{Y}^{Q}=\lambda_{i j}^{U} \bar{Q}_{i} \tilde{\phi}_{1} U_{j}+\lambda_{i j}^{D} \bar{Q}_{i} \phi_{1} D_{j}+\xi_{i j}^{U} \bar{Q}_{i} \tilde{\phi}_{2} U_{j}+\xi_{i j}^{D} \bar{Q}_{i} \phi_{2} D_{j}
$$

where the first two terms give masses of the quark mass eigenstates, and $\xi_{i j}^{U}$ and $\xi_{i j}^{D}$ are the $3 \times 3$ matrices which give the strength of the flavor changing neutral scalar vertices. The $\xi_{\mathrm{s}}$ are all free parameters and can be constrained by the experimental data. If we neglect $\mathrm{CP}$ violation, the $\xi \mathrm{s}$ are 
all real. We will use the Cheng-Sher Ansatz(CSA)[8] and let

$$
\xi_{i j} \sim \frac{\sqrt{m_{i} m_{j}}}{v} .
$$

And we can parametrize the Yukawa couplings as

$$
\xi_{i j}=g \frac{\sqrt{m_{i} m_{j}}}{m_{W}} \lambda .
$$

Comparing it with the usual gauge couplings of $S U(2) \times U(1)$, one has $\lambda=\frac{1}{\sqrt{2}}$. In our calculation we use $\lambda=\frac{1}{\sqrt{2}}$ and note that there is no theoretical bound on the coupling factor $\lambda$.

The subprocess producing $t \bar{c}(\bar{t} c)$ via gluon-gluon collisions,

$$
g g \rightarrow t \bar{c}(\bar{t} c)
$$

can be induced through one-loop diagrams at the lowest order, and the Feynman diagrams are drawn in figure 1(a) and figure 1(b), where the contributions from the one-loop diagrams involving neutral and charged Higgs bosons are presented, respectively. The contributions from the figures of Fig.1(b) with the charged Higgs boson in loops being replaced by W-boson, is much smaller due to the GIM suppression and Yukawa coupling. We can omitted this part in our calculation. The diagrams exchanging the two external gluon-gluon lines are not shown, but are numbered in Fig.1(a) and Fig.1(b). Fig.1(a) $(1 \sim 12)$ and Fig. $1(\mathrm{~b})(1 \sim 6)$ are the self-energy diagrams, Fig. $1(\mathrm{a})(13 \sim 20)$ and Fig. 1 (b) $(7 \sim 10)$ are the vertex correction diagrams, Fig. 1(a) $(25 \sim 34)$ and Fig. 1(b) $(13 \sim 15)$ are the s-channel diagrams, Fig. $1(\mathrm{a})(21 \sim 24)$ and Fig. $1(\mathrm{~b})(11 \sim 12)$ are the box diagrams. Note that in the present case at one-loop level the ultraviolet divergence would be canceled automatically, if all the one-loop diagrams at the $O\left(m_{t} m_{c} / m_{W}^{2}\right)$ order in the THDM III are included. In this work, we perform the calculation in the t'Hooft-Feynman gauge.

To simplify the calculation we set $\alpha=0$ and let all scalar bosons be degenerate, i.e., $m_{h^{0}}=m_{A^{0}}=$ $m_{H^{ \pm}}=M_{s}$ where $M_{s}$ is the common scalar mass. The contribution from the coupling involving $H^{0}$ is suppressed due to $\alpha=0$.

In the calculation for the s-channel diagrams(Fig.1.(a) $(25 \sim 28))$, we take into account the width effects of the $h^{0}$ and $A^{0}$ propagators. The decays of $h^{0}$ to $\mathrm{WW}$ and ZZ are suppressed, because of the factor $\sin \alpha$ in the $h^{0} W W$ and $h^{0} Z Z$ couplings, and $h^{0}$ decay to $A^{0} A^{0}$ is also forbidden due to the case of the degenerate masses of $h^{0}$ and $A^{0}$. Note that the pseudoscalar $A^{0}$ does not couple with gauge boson pair. Therefore only the decays of $h^{0}$ and $A^{0}$ to final states $q_{i} \bar{q}_{j}$ need to be considered, where $q_{i}$ and $q_{j}$ represent quarks of flavor i and $\mathrm{j}$ respectively. The decay width for the scalar $h^{0}$ can be written as [14]

$$
\Gamma\left(h^{0} \rightarrow q \bar{q}\right)=\frac{3 g^{2} m_{h^{0}}}{32 \pi M_{W}^{2}} m_{q}\left(1-4 m_{q}^{2} / m_{h^{0}}^{2}\right)^{3 / 2}
$$

and

$$
\Gamma\left(h^{0} \rightarrow t \bar{c}+\bar{t} c\right)=\frac{3 g^{2} m_{h^{0}}}{32 \pi M_{W}^{2}} \cdot 2 m_{t} m_{c}\left[1-\left(m_{t}+m_{c}\right)^{2} / m_{h^{0}}^{2}\right]^{3 / 2} \times\left[1-\left(m_{t}-m_{c}\right)^{2} / m_{h^{0}}^{2}\right]^{1 / 2} .
$$


The decay width for the pseudoscalar $A^{0}$ boson can be represented by exchanging exponents $3 / 2 \leftrightarrow$ $1 / 2$ and $m_{h^{0}} \leftrightarrow m_{A^{0}}$ in Eq.(5). When $m_{t}+m_{c}<M_{s}<2 m_{t}$, the dominant decay modes of $h^{0}$ and $A^{0}$ are $h^{0}, A^{0} \rightarrow c \bar{c}, b \bar{b}, t \bar{c}+\bar{t} c$, whereas when $M_{s}>2 m_{t}$, the final state $t \bar{t}$ decay channel is open, and their decay widths are rather large due to the large masses of $M_{s}$ and $m_{t}$.

We denote $\theta$ as the scattering angle between one of the gluons and the final top quark. Then we express all the four-momenta of the initial and final particles in the center-of-mass(CMS) by means of the total energy $\sqrt{\hat{s}}$ and the scattering angle $\theta$. The four-momenta of top quark and charm quark are $p_{1}$ and $p_{2}$ respectively and are read

$$
\begin{aligned}
& p_{1}=\left(E_{t}, \sqrt{E_{t}^{2}-m_{t}^{2}} \sin \theta, 0, \sqrt{E_{t}^{2}-m_{t}^{2}} \cos \theta\right), \\
& p_{2}=\left(E_{c},-\sqrt{E_{c}^{2}-m_{c}^{2}} \sin \theta, 0,-\sqrt{E_{c}^{2}-m_{c}^{2}} \cos \theta\right),
\end{aligned}
$$

where

$$
E_{t}=\frac{1}{2}\left(\sqrt{\hat{s}}+\left(m_{t}^{2}-m_{c}^{2}\right) / \sqrt{\hat{s}}\right), \quad E_{c}=\frac{1}{2}\left(\sqrt{\hat{s}}-\left(m_{t}^{2}-m_{c}^{2}\right) / \sqrt{\hat{s}}\right) .
$$

$p_{3}$ and $p_{4}$ are the four-momenta of the initial gluons and are expressed as

$$
p_{3}=\left(\frac{1}{2} \sqrt{\hat{s}}, 0,0, \frac{1}{2} \sqrt{\hat{s}}\right), \quad p_{4}=\left(\frac{1}{2} \sqrt{\hat{s}}, 0,0,-\frac{1}{2} \sqrt{\hat{s}}\right) .
$$

The corresponding matrix element for all the diagrams in figure 1(a) and figure 1(b) is written as

$$
M=\operatorname{Tr}\left(T^{a} T^{b}\right) \delta_{j l} M^{\hat{s}_{1}}+\left(f_{a b c} T_{j l}^{c}\right) M^{\hat{s}_{2}}+\left(T_{j m}^{a} T_{m l}^{b}\right) M^{\hat{t}}+\left(T_{j m}^{b} T_{m l}^{a}\right) M^{\hat{u}}
$$

The upper indexes $\hat{s}_{1}, \hat{s}_{2}, \hat{t}$ and $\hat{u}$ represent the amplitudes corresponding to the s-channel diagrams Fig. 1(a) (25 28), s-channel diagrams Fig. 1(a) $(29 \sim 34)$ and Fig. 1(b) $(13 \sim 15)$, t-channel and u-channel diagrams in figure $1(\mathrm{a})$ and figure $1(\mathrm{~b})$ respectively. The $T^{a}(a=1-8)$ are the $S U(3)_{c}$ generators introduced by Gell-Mann and $f_{a b c}$ are the antisymmetric $S U(3)_{c}$ structure constants. The subscripts $j, l(j, l=1,2,3)$ of $T^{a}$ represent the color of final state top quark and charm quark respectively. The variables $\hat{s}, \hat{t}$ and $\hat{u}$ are usual Mandelstam variables in the center of mass system of gluon-gluon. Their definitions are:

$$
\begin{aligned}
& \hat{s}=\left(p_{1}+p_{2}\right)^{2}=\left(p_{3}+p_{4}\right)^{2}, \quad \hat{t}=\left(p_{1}-p_{3}\right)^{2}=\left(p_{2}-p_{4}\right)^{2}, \\
& \hat{u}=\left(p_{1}-p_{4}\right)^{2}=\left(p_{2}-p_{3}\right)^{2} .
\end{aligned}
$$

We collect all the explicit expressions of the amplitudes appearing in equation (9) in the appendix. The total cross section for $g g \rightarrow t \bar{c}+\bar{t} c$ can be written in the form

$$
\hat{\sigma}(\hat{s})=\frac{2}{16 \pi \hat{s}^{2}} \int_{t^{-}}^{t^{+}} d \hat{t}|\bar{M}|^{2}
$$

where $|\bar{M}|^{2}$ is the initial spin-averaged matrix element squared and $\hat{t}^{ \pm}=1 / 2\left(m_{t}^{2}+m_{c}^{2}-\hat{s}\right) \pm$ $\sqrt{E_{t}^{2}-m_{t}^{2}} \sqrt{\hat{s}}$. The cross section for $p p \rightarrow g g \rightarrow t \bar{c}+\bar{t} c+X$ is conveniently written in terms of the 
rapidities $y_{1}$ and $y_{2}$ of the two jets (finial states) and their common transverse momentum $p_{T}$. Here we neglect the intrinsic transverse momentum carried by partons. It is

$$
\frac{d \sigma}{d y_{1} d y_{2} d p_{T}}=\frac{\pi \tau p_{T}}{\hat{s}} f_{g}\left(x_{1}, Q^{2}\right) f_{g}\left(x_{2}, Q^{2}\right) \hat{\sigma}(g g \rightarrow t \bar{c}+\bar{t} c \text { at } \hat{s}=\tau s)
$$

where $\sqrt{s}$ and $\sqrt{\hat{s}}$ denote the proton-proton and gluon-gluon c.m. energies respectively and $\hat{s}=s \tau$. $f_{g}\left(x_{i}, Q^{2}\right)$ is the distribution function of gluon in proton.

Defining

$$
y^{*}=\frac{1}{2}\left(y_{1}-y_{2}\right)
$$

and

$$
y_{\text {boost }}=\frac{1}{2}\left(y_{1}+y_{2}\right)
$$

We may write

$$
\tau=\frac{4 p_{T}^{2}}{s} \cosh ^{2} y^{*}
$$

and

$$
x_{1}=\sqrt{\tau} e^{y_{\text {boost }}}, \quad x_{2}=\sqrt{\tau} e^{-y_{\text {boost }}} .
$$

In our numerical calculation we adopt the MRS set $\mathrm{G}$ parton distribution function $f_{g}\left(x_{i}, Q^{2}\right)$ 15 and let the factorization scale $Q^{2}=\hat{s}$. The numerical calculation is carried out around the Tevatron and LHC energy ranges.

\section{Numerical Results and Discussions}

In the numerical calculation we take the input parameters [4] as $m_{b}=4.5 \mathrm{GeV}, m_{c}=1.35 \mathrm{GeV}$, $m_{t}=175 \mathrm{GeV}, M_{W}=80.2226 \mathrm{GeV}, G_{F}=1.166392 \times 10^{-5}(\mathrm{GeV})^{-2}$ and $\alpha=1 / 128$. We adopt a simple one-loop formula for the running strong coupling constant $\alpha_{s}$ as

$$
\alpha_{s}(\mu)=\frac{\alpha_{s}\left(m_{Z}\right)}{1+\frac{33-2 n_{f}}{6 \pi} \alpha_{s}\left(m_{Z}\right) \ln \left(\frac{\mu}{m_{Z}}\right)} .
$$

where $\alpha_{s}\left(m_{Z}\right)=0.117$ and $n_{f}$ is the number of active flavors at energy scale $\mu$.

Figure 2 shows the cross sections for $g g \rightarrow t \bar{c}+\bar{t} c$ as a function of the masses of the Higgs bosons $M_{s}$. The cross sections are displayed with the three values of the gluon-gluon CMS energy $200 \mathrm{GeV}$, $400 \mathrm{GeV}$ and $500 \mathrm{GeV}$ respectively. Because there is no stringent bound on the Higgs bosons masses, we choose $M_{s}$ in the range from $50 \mathrm{GeV}$ to $800 \mathrm{GeV}$. The peak of each curve comes from s-channel resonance effects, where $M_{s}=m_{h^{0}}=m_{A^{0}} \sim \sqrt{\hat{s}}$. From these curves we find that the cross section can be obviously enhanced when $M_{s}$ gets close to $\sqrt{\hat{s}}$.

Figure 3 shows the cross sections of $g g \rightarrow t \bar{c}+\bar{t} c$ as a function of $\sqrt{\hat{s}}$, and the three curves correspond to the $M_{s}$ values $100 \mathrm{GeV}, 250 \mathrm{GeV}$ and $500 \mathrm{GeV}$, respectively. For $M_{s}=100 G e V$, 
the effects of the widths of the Higgs bosons are not obvious, and s-channel resonance effects are suppressed, since $\sqrt{\hat{s}}$ is far beyond the Higgs boson masses $M_{s}$. Therefore the curve of its cross section is relative flat with the increasing of $\sqrt{\hat{s}}$. When $\sqrt{\hat{s}}$ approaches the value of $M_{s}$, such as $M_{s}=250 \mathrm{GeV}$ and $M_{s}=500 \mathrm{GeV}$, the cross sections will be enhanced by the s-channel resonance effects, and the width effects become stronger, since the $h^{0}, A^{0} \rightarrow t \bar{c}+\bar{t} c$ channels are opened. In Fig.3, we can see that the curve for $M_{s}=500 \mathrm{GeV}$ shows a sharp peak around the position at $\sqrt{\hat{s}} \simeq 500 \mathrm{GeV}$ due to the s-channel resonance effects and large width effects of $h^{0}$ and $A^{0}$.

In figure 4 and figure 5 we show the transverse momentum spectrum of the top quark at the Tevatron and LHC energies respectively, where we assume the off-line analysis will require at least the event selection criterion of involving one isolated high $p_{T}$ track with the cut of pseudorapidity $|\eta|<2$. Again, the peaks on the curves of $M_{s}=250$ and $M_{s}=500$ show the s-channel resonance effects, where $M_{s}=m_{h^{0}}=m_{A^{0}} \sim \sqrt{\hat{s}}$.

In figure 6 and 7 we show the cross section of $p p \rightarrow g g \rightarrow t \bar{c}+\bar{t} c+X$ as a function of centerof-mass energy of electron-positron system $\sqrt{s}$. The cross section may reach 0.83 femtobarn when $M_{s}=100 \mathrm{GeV}$ and $\sqrt{s}=2 \mathrm{TeV}$ at the Tevatron and 131 femtobarn when $M_{s}=100 \mathrm{GeV}$ and $\sqrt{s}=14 \mathrm{TeV}$ at the LHC. For the Tevatron at $2 \mathrm{TeV}$ we can expect about 4 raw events when $M_{s}=100 \mathrm{GeV}$ if we assume $5 \mathrm{fb}^{-1}$ integrated luminosity, and for the LHC at $14 \mathrm{TeV}$ we can expect about $1.3 \times 10^{4}$ raw events if we assume $100 \mathrm{fb}^{-1}$ integrated luminosity. Since the cross section of this process is roughly scaled by $\lambda^{4}$, if we let $\lambda \simeq 1$, the cross section will be 4 times larger. There are several potentially severe backgrounds from the SM to the signal. A top quark with a mass about $174 G e V$, decays dominantly to $t \rightarrow W^{+} b$. In the $t \bar{c} \rightarrow W^{+} b \bar{c} \rightarrow l^{+} \nu b \bar{c}$ detection mode, the backgrounds are mainly from $t \bar{t} \rightarrow W+$ jets and $t \bar{t} \rightarrow W W b \bar{b} \rightarrow l^{+} \nu q \bar{q}^{\prime} b \bar{b}$ processes. The calculation shows that the cross section of top pair production will reach about $5 p b$ at the Tevatron for $\sqrt{s}=1.8 \mathrm{TeV}$ and about $10^{2} \mathrm{pb}$ at the LHC for $\sqrt{s}=14 \mathrm{TeV}$. If we use the $t \bar{c}+\bar{t} c \rightarrow$ lepton + jets detection mode, the production rate of $t \bar{c} \rightarrow W^{+} b \bar{c} \rightarrow l^{+} \nu b \bar{c}$ can reach about $0.1 \mathrm{fb}$ at the Tevatron and $14 \mathrm{fb}$ at the LHC, while the possible background from $t \bar{t} \rightarrow W W b \bar{b} \rightarrow l^{+} \nu q \bar{q}^{\prime} b \bar{b}$ would be about $0.8 p b$ at the Tevatron and some dozens picobarn at the LHC. The reduction of the these backgrounds is possible through various kinematics cuts on the transverse energy, on the rapidity of jets and leptons, or involving b-tagging. Due to the very small production rate for the signal of $p p \rightarrow t \bar{c}+\bar{t} c$, it is not so easy to suppress its backgrounds. Therefore the further precise analyses are necessary to exclude these backgrounds.

In summary, from our calculation, we can conclude that if we could efficiently exclude the severe backgrounds of the $t \bar{c}(\bar{t} c)$ production signal, it would be possible at the Tevatron and the LHC that the process $p p \rightarrow g g \rightarrow t \bar{c}+\bar{t} c$ could be used to probe the flavor changing interactions in the context of the THDM III.

\section{Acknowledgement:}

This work was supported in part by the National Natural Science Foundation of China(project numbers: 19675033, 19875049), the Youth Science Foundation of the University of Science and Technology of China(USTC) and a grant from the Research Fund for the Doctoral Program of Higher Education(RFDP) of China. 


\section{Appendix}

We adopt the same definitions of one-loop A, B, C and D integral functions as in Ref. [16] and the references therein. The dimension $D=4-\epsilon$. The integral functions are defined as

$$
\begin{gathered}
A_{0}(m)=-\frac{(2 \pi \mu)^{4-D}}{i \pi^{2}} \int d^{D} q \frac{1}{\left[q^{2}-m^{2}\right]}, \\
\left\{B_{1} ; B_{\mu} ; B_{\mu \nu}\right\}\left(p, m_{1}, m_{2}\right)=\frac{(2 \pi \mu)^{4-D}}{i \pi^{2}} \int d^{D} q \frac{\left\{1 ; q_{\mu} ; q_{\mu \nu}\right\}}{\left[q^{2}-m_{1}^{2}\right]\left[(q+p)^{2}-m_{2}^{2}\right]} \\
\left\{C_{0} ; C_{\mu} ; C_{\mu \nu} ; C_{\mu \nu \rho}\right\}\left(p_{1}, p_{2}, m_{1}, m_{2}, m_{3}\right)=-\frac{(2 \pi \mu)^{4-D}}{i \pi^{2}} \\
\times \int d^{D} q \frac{\left\{1 ; q_{\mu} ; q_{\mu \nu} ; q_{\mu \nu \rho}\right\}}{\left[q^{2}-m_{1}^{2}\right]\left[\left(q+p_{1}\right)^{2}-m_{2}^{2}\right]\left[\left(q+p_{1}+p_{2}\right)^{2}-m_{3}^{2}\right]} \\
\left\{D_{0} ; D_{\mu} ; D_{\mu \nu} ; D_{\mu \nu \rho} ; D_{\mu \nu \rho \alpha}\right\}\left(p_{1}, p_{2}, p_{3}, m_{1}, m_{2}, m_{3}, m_{4}\right)=\frac{(2 \pi \mu)^{4-D}}{i \pi^{2}} \\
\times \int d^{D} q\left\{1 ; q_{\mu} ; q_{\mu \nu} ; q_{\mu \nu \rho} ; q_{\mu \nu \rho \alpha}\right\} \\
\left.\times\left\{q^{2}-m_{1}^{2}\right]\left[\left(q+p_{1}\right)^{2}-m_{2}^{2}\right]\left[\left(q+p_{1}+p_{2}\right)^{2}-m_{3}^{2}\right]\left[\left(q+p_{1}+p_{2}+p_{3}\right)^{2}-m_{4}^{2}\right]\right\}^{-1}
\end{gathered}
$$

In our calculation we take the strange quark mass $m_{s}=0$. The $M^{\hat{s}_{1}}$ and $M^{\hat{s}_{2}}$ in equation (9) can be written as

$$
\begin{aligned}
M^{\hat{s}_{1}}= & \frac{i \alpha_{s}^{2} g^{2}}{16 \pi^{2} M_{W}^{2}} m_{t} \sqrt{m_{t} m_{c}} \epsilon_{\mu}\left(p_{3}\right) \epsilon_{\nu}\left(p_{4}\right) \bar{u}\left(p_{1}\right) \\
& \cdot\left\{2 a_{h^{0}} m_{t}^{2}\left(C_{0}+4 C_{22}-4 C_{23}\right)\left[p_{3},-p_{1}-p_{2}, m_{t}, m_{t}, m_{t}\right]\left(p_{1}^{\mu} p_{1}^{\nu}+p_{1}^{\mu} p_{2}^{\nu}+p_{1}^{\nu} p_{2}^{\mu}+p_{2}^{\mu} p_{2}^{\nu}\right)\right. \\
& +2 a_{h^{0}} m_{t}^{2} g^{\mu \nu}\left(B_{0}\left[-p_{1}-p_{2}, m_{t}, m_{t}\right]-\left(\left(p_{1}+p_{2}\right) \cdot p_{3} C_{0}+4 C_{24}\right)\left[p_{3},-p_{1}-p_{2}, m_{t}, m_{t}, m_{t}\right]\right. \\
& \left.+2 i a_{A^{0}} m_{t}^{2} C_{0}\left[p_{3},-p_{1}-p_{2}, m_{t}, m_{t}, m_{t}\right] \epsilon^{\mu \nu \alpha \beta} \gamma_{5}\left(p_{1}^{\alpha} p_{3}^{\beta}+p_{2}^{\alpha} p_{3}^{\beta}\right)\right\} v\left(p_{2}\right)
\end{aligned}
$$

where

$$
a_{h^{0}}=\frac{1}{\hat{s}-m_{h^{0}}^{2}+i \Gamma_{h^{0}} m_{h^{0}}}, \quad a_{A^{0}}=\frac{1}{\hat{s}-m_{A^{0}}^{2}+i \Gamma_{A^{0}} m_{A^{0}}} .
$$




$$
\begin{aligned}
& M^{\hat{s}_{2}}=\frac{i \alpha_{s}^{2} g^{2}}{128 \pi^{2} M_{W}^{2}{ }^{\hat{s}}} m_{t} \sqrt{m_{t} m_{c}} \epsilon_{\mu}\left(p_{3}\right) \epsilon_{\nu}\left(p_{4}\right) \bar{u}\left(p_{1}\right) \\
& \cdot\left\{-16 m_{t}\left(C_{11}-C_{12}+C_{21}-C_{23}\right)\left[-p_{1}, p_{1}+p_{2}, M_{s}, m_{t}, m_{t}\right]\left(p_{1}^{\mu} p_{2}^{\nu}-p_{1}^{\nu} p_{2}^{\mu}\right)\right. \\
& +\left(8\left(2 C_{24}+m_{t}^{2}\left(C_{0}-C_{11}+C_{12}-C_{21}-C_{22}+2 C_{23}\right)-2\left(p_{1} \cdot p_{2}\right)\left(C_{22}+C_{23}\right)\right)\right. \\
& {\left[-p_{1}, p_{1}+p_{2}, M_{s}, m_{t}, m_{t}\right]+4 m_{t}^{2} B_{1}\left[-p_{1}, m_{t}, M_{s}\right]-} \\
& 4 m_{t} m_{c} B_{1}\left[p_{2}, m_{t}, M_{s}\right]+4 m_{t}^{2} B_{1}\left[-p_{1}, m_{b}, M_{s}\right]+ \\
& 4 m_{t} m_{c} B_{1}\left[p_{2}, m_{b}, M_{s}\right]+m_{t}^{2} B_{1}\left[-p_{1}, m_{t}, M_{s}\right] \\
& -m_{t} m_{c} B_{1}\left[p_{2}, m_{t}, M_{s}\right]-m_{t}^{2} B_{1}\left[-p_{1}, m_{b}, M_{s}\right] \\
& \left.+m_{c} m_{t} B_{1}\left[p_{2}, m_{b}, M_{s}\right]\right)\left(\gamma^{\nu} p_{1}^{\mu}-\gamma^{\mu} p_{1}^{\nu}+\gamma^{\nu} p_{2}^{\mu}-\gamma^{\mu} p_{2}^{\nu}+g^{\mu \nu} p_{3}\right) \\
& +\left(-4\left(2 m_{t} C_{24}-2 m_{t}^{3} C_{0}+m_{t}\left(m_{t}^{2}+2\left(p_{1} \cdot p_{2}\right)-4\left(p_{1} \cdot p_{3}\right)\right)\left(C_{11}-C_{12}+C_{21}+C_{22}\right.\right.\right. \\
& \left.\left.-2 C_{23}\right)+4\left(p_{2} \cdot p_{3}\right)\left(m_{c} C_{12}-m_{t} C_{22}+m_{t} C_{23}\right)\right)\left[-p_{1}, p_{1}+p_{2}, M_{s}, m_{t}, m_{t}\right] \\
& -\left(m_{c} m_{t}^{2} C_{0}+2 m_{t} C_{24}+m_{t}^{3}\left(C_{11}-C_{12}+C_{21}+C_{22}-2 C_{23}\right)+2 m_{t}\left(\left(p_{1} \cdot p_{2}\right)-\right.\right. \\
& \left.2\left(p_{1} \cdot p_{2}\right)\right)\left(C_{11}-C_{12}+C_{21}+C_{22}-2 C_{23}\right)+4\left(p_{2} \cdot p_{3}\right)\left(m_{c} C_{12}\right. \\
& \left.+m_{t}\left(C_{23}-C_{22}\right)\right)\left[-p_{1}, p_{1}+p_{2}, M_{s}, m_{b}, m_{b}\right]-m_{t}^{3} B_{1}\left[-p_{1}, m_{t}, M_{s}\right]+ \\
& m_{t}^{2} m_{c} B_{1}\left[p_{2}, m_{t}, M_{s}\right]-m_{t}^{3} B_{1}\left[-p_{1}, m_{b}, M_{s}\right]+\left(m_{c} m_{t}^{2}+\right. \\
& \left.\left.2 m_{c} p_{1} \cdot p_{2}-4 m_{c} p_{1} \cdot p_{3}\right) B_{1}\left[p_{2}, m_{b}, M_{s}\right]\right) g^{\mu \nu}-4 m_{t}\left(C_{11}\right. \\
& \left.-C_{12}+C_{21}-C_{23}\right)\left[-p_{1}, p_{1}+p_{2}, M_{s}, m_{b}, m_{b}\right]\left(p_{1}^{\mu} p_{2}^{\nu}-p_{1}^{\nu} p_{2}^{\mu}+\gamma_{5} p_{1}^{\nu} p_{2}^{\mu}-\gamma_{5} p_{1}^{\mu} p_{2}^{\nu}\right) \\
& +\left(2\left(2 C_{24}+m_{c} m_{t} C_{0}-m_{t}^{2}\left(C_{11}-C_{12}+C_{21}+C_{22}-2 C_{23}\right)-2\left(p_{1} \cdot p_{2}\right)\left(C_{22}-C_{23}\right)\right)\right. \\
& {\left[-p_{1}, p_{1}+p_{2}, M_{s}, m_{b}, m_{b}\right]+4 m_{t}^{2} B_{1}\left[-p_{1}, m_{b}, M_{s}\right]-} \\
& \left.4 m_{c} m_{t} B_{1}\left[p_{2}, m_{b}, M_{s}\right]\right)\left(\gamma^{\nu} p_{1}^{\mu}-\gamma^{\mu} p_{1}^{\nu}+\gamma^{\nu} p_{2}^{\mu}-\gamma^{\mu} p_{2}^{\nu}+g^{\mu \nu} p_{3}\right)-\left(m_{c} m_{t}^{2} C_{0}-2 m_{t} C_{24}\right. \\
& -m_{t}^{3}\left(C_{11}-C_{12}+C_{21}+C_{22}-2 C_{23}\right)-2 m_{t}\left(\left(p_{1} \cdot p_{2}\right)-2\left(p_{1} \cdot p_{2}\right)\right)\left(C_{11}-C_{12}+C_{21}\right. \\
& \left.+C_{22}-2 C_{23}\right)+4\left(p_{2} \cdot p_{3}\right)\left(m_{c} C_{12}-m_{t}\left(C_{23}-C_{22}\right)\right)\left[-p_{1}, p_{1}+p_{2}, M_{s}, m_{b}, m_{b}\right] \gamma_{5} g^{\mu \nu} \\
& +\left(\left(4 C_{24}-2 m_{c} m_{t} C_{0}-2 m_{t}^{2}\left(C_{11}-C_{12}+C_{21}+C_{22}-2 C_{23}\right)+4\left(p_{1} \cdot p_{2}\right)\left(C_{23}\right.\right.\right. \\
& \left.\left.\left.-C_{22}\right)\right)\left[-p_{1}, p_{1}+p_{2}, M_{s}, m_{b}, m_{b}\right]\right)+2 m_{t}^{2} B_{1}\left[-p_{1}, m_{b}, M_{s}\right]- \\
& \left.\left.2 m_{c} m_{t} B_{1}\left[p_{2}, m_{b}, M_{s}\right]\right) \gamma_{5}\left(\gamma^{\nu} p_{1}^{\mu}-\gamma^{\mu} p_{1}^{\nu}+\gamma^{\nu} p_{2}^{\mu}-\gamma^{\mu} p_{2}^{\nu}-g^{\mu \nu} p_{3}\right)\right\} v\left(p_{2}\right),
\end{aligned}
$$

The amplitude of $M^{\hat{t}}$ can be written as

$$
\begin{aligned}
M^{\hat{t}}= & \frac{i \alpha_{s}^{2} g^{2}}{64 \pi^{2} M_{W}^{2}} m_{t} \sqrt{m_{t} m_{c}} \epsilon_{\mu}\left(p_{3}\right) \epsilon_{\nu}\left(p_{4}\right) \bar{u}\left(p_{1}\right)\left(f_{1} p_{1}^{\mu} p_{1}^{\nu}+f_{2} p_{1}^{\mu} p_{2}^{\nu}+f_{3} p_{1}^{\nu} p_{2}^{\mu}+f_{4} p_{2}^{\mu} p_{2}^{\nu}\right. \\
& +f_{5} \gamma^{\nu} p_{1}^{\mu}+f_{6} \gamma^{\mu} p_{1}^{\nu}+f_{7} \gamma^{\nu} p_{2}^{\mu}+f_{8} \gamma^{\mu} p_{2}^{\nu}+f_{9} \gamma^{\mu} \gamma^{\nu}+f_{10} \gamma^{\nu} \gamma^{\mu}+f_{11} p_{3} p_{1}^{\mu} p_{1}^{\nu} \\
& +f_{12} p_{3} p_{1}^{\mu} p_{2}^{\nu}+f_{13} p_{3} p_{1}^{\nu} p_{2}^{\mu}+f_{14} p_{3} p_{2}^{\mu} p_{2}^{\nu}+f_{15} p_{3} \gamma^{\nu} p_{1}^{\mu}+f_{16} p_{3} \gamma^{\mu} p_{1}^{\nu}+f_{17} p_{3} \gamma^{\nu} p_{2}^{\mu} \\
& +f_{18} p_{3} \gamma^{\mu} p_{2}^{\nu}+f_{19} p_{3} \gamma^{\mu} \gamma^{\nu}+f_{20} p_{3} \gamma^{\nu} \gamma^{\mu}+f_{1}^{\prime} \gamma_{5} p_{1}^{\mu} p_{1}^{\nu}+f_{2}^{\prime} \gamma_{5} p_{1}^{\mu} p_{2}^{\nu}+f_{3}^{\prime} \gamma_{5} p_{1}^{\nu} p_{2}^{\mu} \\
& +f_{4}^{\prime} \gamma_{5} p_{2}^{\mu} p_{2}^{\nu}+f_{5}^{\prime} \gamma_{5} \gamma^{\nu} p_{1}^{\mu}+f_{6}^{\prime} \gamma_{5} \gamma^{\mu} p_{1}^{\nu}+f_{7}^{\prime} \gamma_{5} \gamma^{\nu} p_{2}^{\mu}+f_{8}^{\prime} \gamma_{5} \gamma^{\mu} p_{2}^{\nu}+f_{9}^{\prime} \gamma_{5} \gamma^{\mu} \gamma^{\nu} \\
& +f_{10}^{\prime} \gamma_{5} \gamma^{\nu} \gamma^{\mu}+f_{11}^{\prime} \gamma_{5} p_{3} p_{1}^{\mu} p_{1}^{\nu}+f_{12}^{\prime} \gamma_{5} p_{3} p_{1}^{\mu} p_{2}^{\nu}+f_{13}^{\prime} \gamma_{5} p_{3} p_{1}^{\nu} p_{2}^{\mu}+f_{14}^{\prime} \gamma_{5} p_{3} p_{2}^{\mu} p_{2}^{\nu} \\
& +f_{15}^{\prime} \gamma_{5} p_{3} \gamma^{\nu} p_{1}^{\mu}+f_{16}^{\prime} \gamma_{5} p_{3} \gamma^{\mu} p_{1}^{\nu}+f_{17}^{\prime} \gamma_{5} p_{3} \gamma^{\nu} p_{2}^{\mu}+f_{18}^{\prime} \gamma_{5} p_{3} \gamma^{\mu} p_{2}^{\nu} \\
& \left.+f_{19}^{\prime} \gamma_{5} p_{3} \gamma^{\mu} \gamma^{\nu}+f_{20}^{\prime} \gamma_{5} p_{3} \gamma^{\nu} \gamma^{\mu}\right) v\left(p_{2}\right),
\end{aligned}
$$

where the $f_{i} s$ and $f_{i}^{\prime} s$ are expressed explicitly as,

$$
f_{1}^{\prime}=4\left(m_{c}\left(D_{38}-D_{310}\right)+m_{t}\left(D_{32}-D_{36}\right)\right)\left[p_{3},-p_{1},-p_{2}, m_{b}, m_{b}, M_{s}, m_{b}\right]
$$




$$
f_{1}=f_{1}^{\prime}\left(m_{t} \rightarrow-m_{t}\right)+8 m_{t}\left(D_{11}-D_{12}+D_{21}-D_{24}-D_{25}+D_{26}\right)\left[p_{1},-p_{3},-p_{4}, M_{s}, m_{t}, m_{t}, m_{t}\right]
$$

$$
\begin{aligned}
& f_{2}^{\prime}= 4 a_{1}\left(m_{c} C_{22}-m_{t} C_{12}-m_{t} C_{23}\right)\left[-p_{4}, p_{2}, m_{b}, m_{b}, M_{s}\right] \\
&+4\left(m_{c}\left(D_{39}-D_{310}\right)-m_{t}\left(D_{22}-D_{26}+D_{36}-D_{38}\right)\right)\left[p_{3},-p_{1},-p_{2}, m_{b}, m_{b}, M_{s}, m_{b}\right] \\
& f_{2}= \\
& \quad f_{2}^{\prime}\left(m_{t} \rightarrow-m_{t}\right)+8 a_{1} m_{t} C_{11}\left[-p_{2}, p_{4}, M_{s}, m_{t}, m_{t}\right] \\
&-8 m_{t}\left(D_{12}+D_{24}-D_{26}\right)\left[p_{1},-p_{3},-p_{4}, M_{s}, m_{t}, m_{t}, m_{t}\right] \\
& f_{3}^{\prime}= 4\left(m_{c}\left(D_{39}-D_{37}\right)-m_{t}\left(D_{310}-D_{38}\right)\right)\left[p_{3},-p_{1},-p_{2}, m_{b}, m_{b}, M_{s}, m_{b}\right] \\
& f_{3}=f_{3}^{\prime}\left(m_{t} \rightarrow-m_{t}\right)-8 m_{t}\left(D_{25}-D_{26}\right)\left[p_{1},-p_{3},-p_{4}, M_{s}, m_{t}, m_{t}, m_{t}\right] \\
& f_{4}^{\prime}= 4\left(m_{c}\left(D_{33}-D_{37}\right)+m_{t}\left(D_{23}-D_{26}-D_{310}+D_{39}\right)\right)\left[p_{3},-p_{1},-p_{2}, m_{b}, m_{b}, M_{s}, m_{b}\right] \\
& \\
& f_{4}=f_{4}^{\prime}\left(m_{t} \rightarrow-m_{t}\right)+8 m_{t} D_{26}\left[p_{1},-p_{3},-p_{4}, M_{s}, m_{t}, m_{t}, m_{t}\right] \\
& f_{5}^{\prime}= \\
&-2 a_{2} a_{3} m_{t}^{2} B_{1}\left[-p_{1}, m_{b}, M_{s}\right]-2 a_{1} a_{2}\left(m_{t}^{2}-2\left(p_{1} \cdot p_{3}\right)\right) B_{1}\left[-p_{1}+p_{3}, m_{b}, M_{s}\right] \\
&-2 a_{1} a_{3} m_{c} m_{t} B_{1}\left[p_{2}, m_{b}, M_{s}\right]+2 a_{2}\left(m_{c} m_{t}\left(C_{0}+2 C_{11}+C_{21}\right)-2 C_{24}\right)\left[p_{1},-p_{3}, M_{s}, m_{t}, m_{t}\right] \\
&+2 a_{1}\left(-2 C_{24}-m_{b}^{2} C_{0}+m_{c}^{2}\left(C_{22}-2 C_{23}\right)-m_{c} m_{t} C_{12}+m_{t}^{2}\left(C_{11}+C_{21}\right)\right. \\
&+2\left(p_{1} \cdot p_{2}-p_{2} \cdot p_{3}\right)\left(C_{11}-C_{12}+C_{21}-C_{23}\right) \\
&\left.-2\left(p_{1} \cdot p_{3}\right)\left(C_{11}+C_{21}\right)\right)\left[-p_{4}, p_{2}, m_{b}, m_{b}, M_{s}\right] \\
&+2\left(2 D_{27}+2 D_{312}+m_{b}^{2} D_{0}-m_{c}^{2} D_{23}-m_{c} m_{t}\left(D_{22}-D_{26}\right)\right. \\
&\left.+2\left(p_{2} \cdot p_{3}\right)\left(D_{25}-D_{26}\right)\right)\left[p_{3},-p_{1},-p_{2}, m_{b}, m_{b}, M_{s}, m_{b}\right]
\end{aligned}
$$

$$
\begin{aligned}
f_{5}= & f_{5}^{\prime}\left(m_{t} \rightarrow-m_{t}\right)+4 a_{2} a_{3} m_{t}^{2} B_{0}\left[-p_{1}, m_{t}, M_{s}\right] \\
& +4 a_{1} a_{2} m_{t}^{2} B_{0}\left[-p_{1}+p_{3}, m_{t}, M_{s}\right]-4 a_{1} a_{3} m_{t}^{2} B_{0}\left[p_{2}, m_{t}, M_{s}\right] \\
& -4 a_{2} m_{t}^{2}\left(C_{0}+C_{11}\right)\left[p_{1},-p_{3}, M_{s}, m_{t}, m_{t}\right]-4 a_{1} m_{t}^{2}\left[-p_{2}, p_{4}, M_{s}, m_{t}, m_{t}\right] \\
& +4 m_{t}^{2}\left(D_{0}+D_{11}-D_{13}\right)\left[p_{1},-p_{3},-p_{4}, M_{s}, m_{t}, m_{t}, m_{t}\right]
\end{aligned}
$$

$$
\begin{aligned}
f_{6}^{\prime}= & 2\left(4\left(D_{311}-D_{312}\right)+m_{b}^{2}\left(D_{11}-D_{12}\right)-m_{c}^{2}\left(D_{37}-D_{39}\right)\right. \\
& +m_{t}^{2}\left(D_{32}-D_{36}\right)+2\left(p_{1} \cdot p_{2}\right)\left(D_{38}-D_{310}\right)-2\left(p_{1} \cdot p_{3}\right)\left(D_{22}-D_{24}-D_{34}+D_{36}\right) \\
& \left.+2\left(p_{1} \cdot p_{2}\right)\left(D_{35}-D_{310}\right)\right)\left[p_{3},-p_{1},-p_{2}, m_{b}, m_{b}, M_{s}, m_{b}\right]
\end{aligned}
$$

$$
f_{6}=f_{6}^{\prime}
$$




$$
\begin{aligned}
& f_{7}^{\prime}=2\left(-4 D_{313}-m_{b}^{2} D_{13}+m_{c}^{2} D_{33}+m_{c} m_{t} D_{23}+m_{t}^{2}\left(D_{26}+D_{38}\right)+2\left(p_{1} \cdot p_{2}\right)\left(D_{23}+D_{39}\right)\right. \\
& \left.-2\left(p_{1} \cdot p_{3}\right)\left(D_{25}+D_{310}+D_{23}+D_{37}\right)\right)\left[p_{3},-p_{1},-p_{2}, m_{b}, m_{b}, M_{s}, m_{b}\right] \\
& f_{7}=f_{7}^{\prime}\left(m_{t} \rightarrow-m_{t}\right)-4 m_{t}^{2} D_{13}\left[p_{1},-p_{3},-p_{4}, M_{s}, m_{t}, m_{t}, m_{t}\right] \\
& f_{8}^{\prime}=-4 a_{1}\left(p_{1} \cdot p_{3}\right)\left(C_{12}+C_{23}\right)\left[-p_{4}, p_{2}, m_{b}, m_{b}, M_{s}\right] \\
& +2\left(2\left(D_{27}+2 D_{311}+D_{313}\right)+m_{b}^{2}\left(D_{0}+D_{11}\right)-m_{c}^{2}\left(D_{23}+D_{37}\right)\right. \\
& -m_{t}^{2}\left(D_{22}+D_{36}\right)\left[p_{3},-p_{1},-p_{2}, m_{b}, m_{b}, M_{s}, m_{b}\right] \\
& -\left(p_{1} \cdot p_{2}\right)\left(D_{26}+D_{310}\right)+2\left(p_{1} \cdot p_{3}\right)\left(D_{12}-D_{13}+4 D_{24}-D_{26}+D_{34}\right) \\
& \left.+2\left(p_{2} \cdot p_{3}\right)\left(D_{25}+D_{35}\right)\right)\left[p_{3},-p_{1},-p_{2}, m_{b}, m_{b}, M_{s}, m_{b}\right] \\
& f_{8}=f_{8}^{\prime} \\
& f_{9}^{\prime}=-a_{2} a_{3} m_{t}^{3} B_{1}\left[-p_{1}, m_{b}, M_{s}\right] \\
& +2 a_{1} a_{2} m_{c}\left(p_{1} \cdot p_{3}\right) B_{1}\left[-p_{1}+p_{3}, m_{b}, M_{s}\right]-a_{2} m_{t}\left(2 C_{24}\right. \\
& \left.-m_{t}^{2}\left(C_{0}+2 C_{11}+C_{21}\right)+2\left(p_{1} \cdot p_{3}\right)\left(C_{0}+C_{11}+C_{12}+C_{23}\right)\right)\left[p_{1},-p_{3}, M_{s}, m_{t}, m_{t}\right] \\
& -2 a_{1} m_{c}\left(p_{1} \cdot p_{3}\right) C_{12}\left[-p_{4}, p_{2}, m_{b}, m_{b}, M_{s}\right]+\left(4 m _ { c } \left(D_{313}\right.\right. \\
& \left.+m_{b}^{2} D_{13}-m_{c}^{2} D_{33}\right)+m_{t}\left(2 D_{27}+4 D_{312}+m_{b}^{2}\left(D_{0}+D_{12}\right)-m_{c}^{2}\left(D_{23}+D_{39}\right)\right. \\
& \left.-m_{t}\left(m_{c} D_{38}-m_{t} D_{22}-m_{t} D_{32}\right)\right)-2\left(p_{1} \cdot p_{2}\right)\left(m_{c} D_{39}\right. \\
& \left.+m_{t} D_{26}+m_{t} D_{38}\right)+2\left(p_{1} \cdot p_{3}\right)\left(m_{c} D_{12}-m_{c} D_{13}+m_{c} D_{310}+m_{t} D_{24}+m_{t} D_{36}\right) \\
& \left.+2\left(p_{2} \cdot p_{3}\right)\left(m_{c} D_{37}+m_{t} D_{25}+m_{t} D_{310}\right)\right)\left[p_{3},-p_{1},-p_{2}, m_{b}, m_{b}, M_{s}, m_{b}\right] \\
& f_{9}=f_{9}^{\prime}\left(m_{t} \rightarrow-m_{t}\right)-2 a_{2} a_{3} m_{t}^{3} B_{0}\left[-p_{1}, m_{t}, M_{s}\right] \\
& -4 a_{1} a_{2} m_{t}\left(p_{1} \cdot p_{3}\right) B_{0}\left[-p_{1}+p_{3}, m_{t}, M_{s}\right] \\
& +4 a_{2} m_{t}\left(p_{1} \cdot p_{3}\right) C_{0}\left[p_{1},-p_{3}, M_{s}, m_{t}, m_{t}\right] \\
& +4 a_{1} m_{t}\left(p_{1} \cdot p_{3}\right) C_{0}\left[-p_{2}, p_{4}, M_{s}, m_{t}, m_{t}\right] \\
& +4 m_{t}\left(D_{27}+D_{311}-D_{313}\right)\left[p_{1},-p_{3},-p_{4}, M_{s}, m_{t}, m_{t}, m_{t}\right] \\
& f_{10}^{\prime}=-2\left(m_{c} D_{313}+m_{t} D_{312}\right)\left[p_{3},-p_{1},-p_{2}, m_{b}, m_{b}, M_{s}, m_{b}\right] \\
& f_{10}=f_{10}^{\prime}\left(m_{t} \rightarrow-m_{t}\right)-4 m_{t} D_{27}\left[p_{1},-p_{3},-p_{4}, M_{s}, m_{t}, m_{t}, m_{t}\right] \\
& f_{11}^{\prime}=4\left(D_{22}-D_{24}-D_{34}+D_{36}\right)\left[p_{3},-p_{1},-p_{2}, m_{b}, m_{b}, M_{s}, m_{b}\right] \\
& f_{11}=f_{11}^{\prime} \\
& f_{12}^{\prime}=4\left(D_{13}-D_{12}-2 D_{24}+2 D_{26}+D_{310}-D_{34}\right)\left[p_{3},-p_{1},-p_{2}, m_{b}, m_{b}, M_{s}, m_{b}\right]
\end{aligned}
$$




$$
\begin{aligned}
& f_{12}=f_{12}^{\prime} \\
& f_{13}^{\prime} \\
& f_{13}=f_{13}^{\prime} \\
& f_{14}^{\prime}=4\left(D_{23}-D_{25}-D_{35}+D_{37}\right)\left[p_{3},-p_{1},-p_{2}, m_{b}, m_{b}, M_{s}, m_{b}\right] \\
& f_{14}=f_{14}^{\prime} \\
& f_{15}^{\prime}=-2 a_{2} m_{t}\left(C_{11}-C_{12}+C_{21}-C_{23}\right)\left[p_{1},-p_{3}, M_{s}, m_{t}, m_{t}\right] \\
& +2\left(m_{c}\left(D_{13}-D_{12}+D_{26}\right)+m_{t}\left(D_{22}-D_{24}\right)\right)\left[p_{3},-p_{1},-p_{2}, m_{b}, m_{b}, M_{s}, m_{b}\right] \\
& f_{15}=f_{15}^{\prime}\left(m_{t} \rightarrow-m_{t}\right)+4 a_{2} m_{t} C_{11}\left[p_{1},-p_{3}, M_{s}, m_{t}, m_{t}\right] \\
& -4 m_{t}\left(D_{11}-D_{13}\right)\left[p_{1},-p_{3},-p_{4}, M_{s}, m_{t}, m_{t}, m_{t}\right] \\
& f_{16}^{\prime}=2\left(m_{c}\left(D_{25}-D_{26}\right)-m_{t}\left(D_{22}-D_{24}\right)\right)\left[p_{3},-p_{1},-p_{2}, m_{b}, m_{b}, M_{s}, m_{b}\right] \\
& f_{16}=f_{16}^{\prime}\left(m_{t} \rightarrow-m_{t}\right)+4 m_{t}\left(D_{11}-D_{12}\right)\left[p_{1},-p_{3},-p_{4}, M_{s}, m_{t}, m_{t}, m_{t}\right] \\
& f_{17}^{\prime}=2\left(m_{c} D_{23}-m_{t}\left(D_{25}-D_{26}\right)\right)\left[p_{3},-p_{1},-p_{2}, m_{b}, m_{b}, M_{s}, m_{b}\right] \\
& f_{17}=f_{17}^{\prime}\left(m_{t} \rightarrow-m_{t}\right)+4 m_{t} D_{13}\left[p_{1},-p_{3},-p_{4}, M_{s}, m_{t}, m_{t}, m_{t}\right] \\
& f_{18}^{\prime}=2 a_{1}\left(m_{c} C_{22}-m_{t} C_{12}-m_{t} C_{23}\right)\left[-p_{4}, p_{2}, m_{b}, m_{b}, M_{s}\right] \\
& +2\left(m_{c}\left(D_{25}-D_{23}\right)+m_{t}\left(D_{12}-D_{13}+D_{24}-D_{26}\right)\right)\left[p_{3},-p_{1},-p_{2}, m_{b}, m_{b}, M_{s}, m_{b}\right] \\
& f_{18}=f_{18}^{\prime}\left(m_{t} \rightarrow-m_{t}\right)+4 a_{1} m_{t} C_{11}\left[-p_{2}, p_{4}, M_{s}, m_{t}, m_{t}\right] \\
& -4 m_{t} D_{12}\left[p_{1},-p_{3},-p_{4}, M_{s}, m_{t}, m_{t}, m_{t}\right]
\end{aligned}
$$




$$
\begin{aligned}
& f_{19}^{\prime}=-a_{2} a_{3} m_{t}^{2} B_{1}\left[-p_{1}, m_{b}, M_{s}\right] \\
&-a_{1} a_{2}\left(m_{t}^{2}-2\left(p_{1} \cdot p_{3}\right)\right) B_{1}\left[-p_{1}+p_{3}, m_{b}, M_{s}\right] \\
&-a_{1} a_{3} m_{c} m_{t} B_{1}\left[p_{2}, m_{b}, M_{s}\right]-a_{2}\left(2 C_{24}-m_{t}\left(m_{c} C_{0}+m_{t} C_{11}\right.\right. \\
&\left.\left.+m_{t} C_{21}\right)+2\left(C_{12}+C_{23}\right)\left(p_{1} \cdot p_{3}\right)\right)\left[p_{1},-p_{3}, M_{s}, m_{t}, m_{t}\right] \\
&+a_{1}\left(-2 C_{24}-m_{b}^{2} C_{0}+m_{c}^{2}\left(C_{22}-2 C_{23}\right)-m_{c} m_{t} C_{12}\right. \\
&+m_{t}^{2}\left(C_{11}+C_{21}\right)+2\left(\left(p_{1} \cdot p_{2}\right)-\left(p_{2} \cdot p_{3}\right)\right)\left(C_{11}-C_{12}+C_{21}-C_{23}\right) \\
&-2\left(p_{1} \cdot p_{3}\right)\left(C_{11}+C_{21}\right)\left[-p_{4}, p_{2}, m_{b}, m_{b}, M_{s}\right] \\
&+\left(4\left(D_{27}+D_{311}\right)+2 m_{b}^{2}\left(D_{0}+D_{11}\right)-2 m_{c}^{2}\left(D_{23}+D_{37}\right)-D_{13} m_{c} m_{t}\right. \\
&-m_{t}^{2}\left(D_{12}+D_{22}+D_{36}\right)-2\left(p_{1} \cdot p_{2}\right)\left(D_{12}+2 D_{26}+D_{310}\right)+2\left(p_{1} \cdot p_{3}\right)\left(D_{12}\right. \\
&\left.\left.+2 D_{24}+D_{34}\right)+2\left(p_{2} \cdot p_{3}\right)\left(D_{13}+2 D_{25}+D_{35}\right)\right)\left[p_{3},-p_{1},-p_{2}, m_{b}, m_{b}, M_{s}, m_{b}\right] \\
& \quad f_{19}=\quad f_{19}^{\prime}\left(m_{t} \rightarrow-m_{t}\right)+2 a_{2} a_{3} m_{t}^{2} B_{0}\left[-p_{1}, m_{t}, M_{s}\right] \\
&+2 a_{1} a_{2} m_{t}^{2} B_{0}\left[-p_{1}+p_{3}, m_{t}, M_{s}\right]-2 a_{1} a_{2} m_{t}^{2} B_{0}\left[p_{2}, m_{t}, M_{s}\right] \\
&-2 a_{2} m_{t}^{2} C_{0}\left[p_{1},-p_{3}, M_{s}, m_{t}, m_{t}\right]-2 a_{1} m_{t}^{2} C_{0}\left[-p_{2}, p_{4}, M_{s}, m_{t}, m_{t}\right] \\
&+2 m_{t}^{2} D_{0}\left[p_{1},-p_{3},-p_{4}, M_{s}, m_{t}, m_{t}, m_{t}\right] \\
& f_{20}^{\prime}=-2 D_{311}\left[p_{3},-p_{1},-p_{2}, m_{b}, m_{b}, M_{s}, m_{b}\right] \\
& \quad f_{20}=f_{20}^{\prime}
\end{aligned}
$$

where

$$
\begin{aligned}
& a_{1}=\frac{1}{\hat{t}-m_{t}^{2}}, \quad a_{2}=\frac{1}{\hat{t}-m_{c}^{2}} \text { and } a_{3}=\frac{1}{m_{t}^{2}-m_{c}^{2}} . \\
& M^{\hat{u}}=M^{\hat{t}} \quad\left(p_{3} \leftrightarrow p_{4}, \quad \mu \leftrightarrow \nu, \hat{t} \leftrightarrow \hat{u}\right) \quad(A .44)
\end{aligned}
$$

\section{References}

[1] S. Glashow and S. Weinberg, Phys. Rev. D15, 1958(1977) ; T. P. Cheng and M. Sher, ibid. D35, 3484(1987); M. Sher and Y. Yuan, ibid. 44, 1461(1991).

[2] M. Luke and M. J. Savage, Phys. Lett. B207, 387(1993).

[3] S. L. Glashow and S. Weinberg, Phys. Rev. D15, 1958(1977).

[4] C. Caso, G. Conforto et al., 'Review of Particle Physics', Euro. Phys. J. C3, (1998)1.

[5] F. Abe et al., CDF Collaboration, Phys. Rev. Lett. 80, 2779(1998); F. Abe et al., CDF Collaboration, Phys. Rev. Lett. 80, 2767(1998); S. Abachi et al., The D0 Collaboration, Phys. Rev. Lett. 79, 1197(1997). 
[6] A. Antaramian, L. J. Hall and A. Rasin, Phys. Rev. Lett.69, 1871(1992).

[7] D. Chang, W. S. Hou and W. Y. Keung, CERN preprint CERN-TH. 6795/93(1993).

[8] T. P. Cheng and M. Sher, Phys. Rev. D35, 3484(1987); M. Sher and Y. Yuan, ibid. 44, 1461(1991)

[9] W. S. Hou, Phys. Lett. B 296, 179(1992); D. Chang, W. S. Hou and W. Y. Keung, Phys. Rev. D48,217(1993).

[10] David Atwood et al. Phys. Rev. D53, 1199(1996).

[11] Jiang Yi, Zhou Mian-Lai, Ma Wen-Gan, Han Liang, Zhou Hong and Han Meng, Phys. Rev. D57, 4343(1998);

[12] K. J. Abraham et al., Phys. Lett. B419, 381(1998).

[13] Wei-Shu Hou and Guey-Lin Lin, Phys. Lett. B379, 261(1996).

[14] W. S. Hou, Phys. Lett. B 296, 179(1992).

[15] A.D. Martin, W.J. Stirling and R.G. Roberts, Phys. Lett.B354, 155(1995).

[16] Kniehl B. A., Phys. Rep. 240(1994)211 and references therein.

\section{Figure Captions}

Fig.1(a)(b) The Feynman diagrams of the subprocess $g g \rightarrow t \bar{c}$.

Fig.2 Total cross sections of the subprocess $g g \rightarrow t \bar{c}+\bar{t} c$ as function of $M_{s}$. The solid curve is for $\sqrt{\hat{s}}=200 \mathrm{GeV}$, the dashed curve is for $\sqrt{\hat{s}}=400 \mathrm{GeV}$ and the dotted curve is for $\sqrt{\hat{s}}=500 \mathrm{GeV}$.

Fig.3 Total cross sections of the subprocess $g g \rightarrow t \bar{c}+\bar{t} c$ as function of $\sqrt{\hat{s}}$. The solid curve is for $M_{s}=100 \mathrm{GeV}$, the dashed curve is for $M_{s}=250 \mathrm{GeV}$ and the dotted curve is for $M_{s}=500 \mathrm{GeV}$.

Fig.4 The transverse momentum spectrum of top quark for the Tevatron at $2 \mathrm{TeV}$. The solid curve is for $M_{s}=100 \mathrm{GeV}$, the dashed curve is for $M_{s}=250 \mathrm{GeV}$ and the dotted curve is for $M_{s}=500 \mathrm{GeV}$.

Fig.5 The transverse momentum spectrum of top quark for the LHC at $14 \mathrm{TeV}$. The solid curve is for $M_{s}=100 \mathrm{GeV}$, the dashed curve is for $M_{s}=250 \mathrm{GeV}$ and the dotted curve is for $M_{s}=500 \mathrm{GeV}$.

Fig.6 Total cross sections of the process $p p \rightarrow g g \rightarrow t \bar{c}+\bar{t} c$ as function of $\sqrt{s}$ at the Tevatron. The solid curve is for $M_{s}=100 \mathrm{GeV}$, the dashed curve is for $M_{s}=250 \mathrm{GeV}$ and the dotted curve is for $M_{s}=500 \mathrm{GeV}$.

Fig.7 Total cross sections of the process $p p \rightarrow g g \rightarrow t \bar{c}+\bar{t} c$ as function of $\sqrt{s}$ at the LHC. The solid curve is for $M_{s}=100 \mathrm{GeV}$, the dashed curve is for $M_{s}=250 \mathrm{GeV}$ and the dotted curve is for $M_{s}=500 \mathrm{GeV}$. 


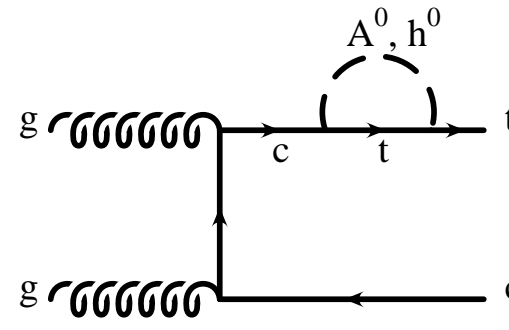

$(1,2,3,4)$

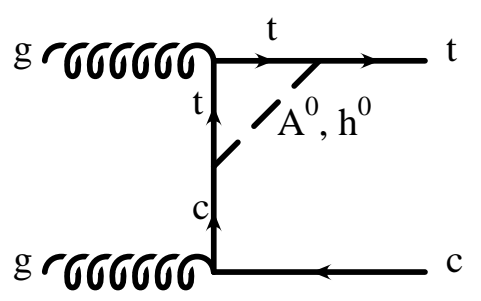

$(13,14,15,16)$

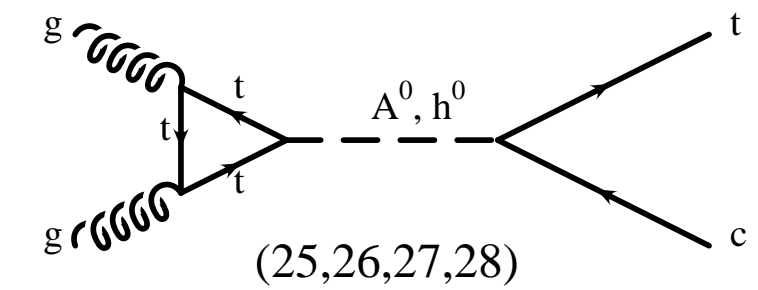

$(31,32)$

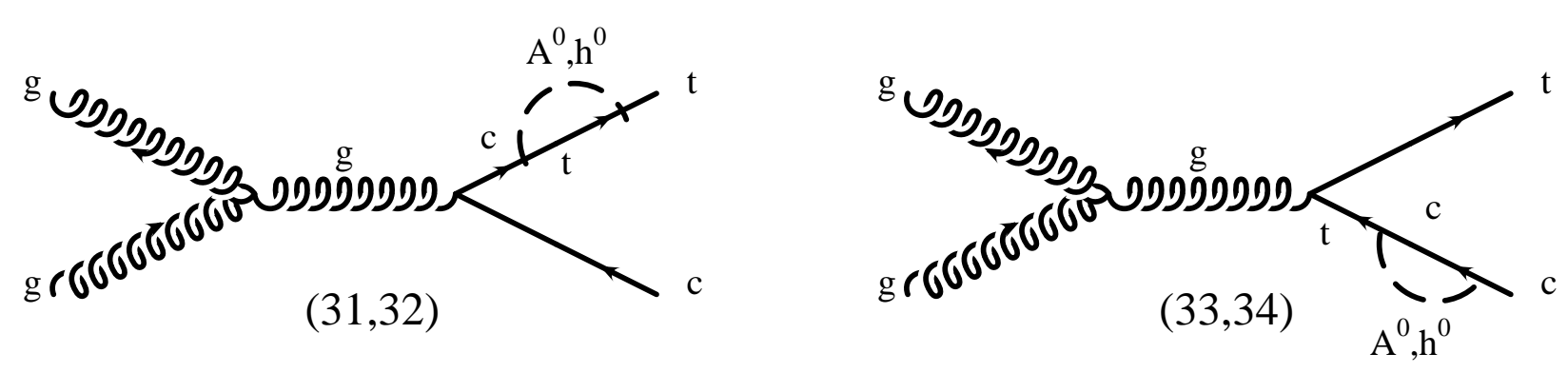

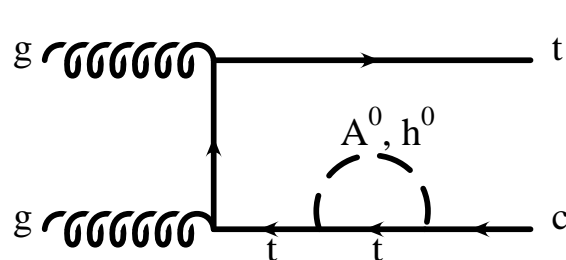

$(5,6,7,8)$

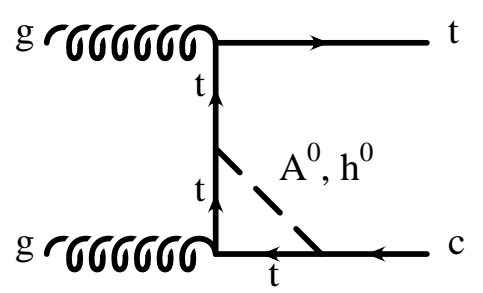

$(17,18,19,20)$

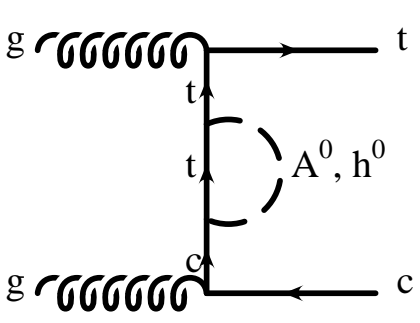

$(9,10,11,12)$

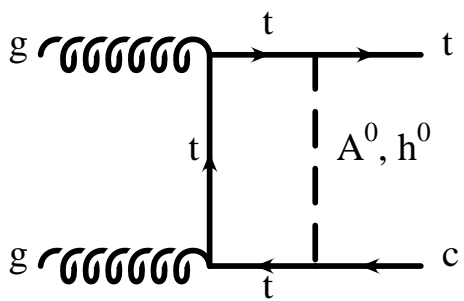

$(21,22,23,24)$

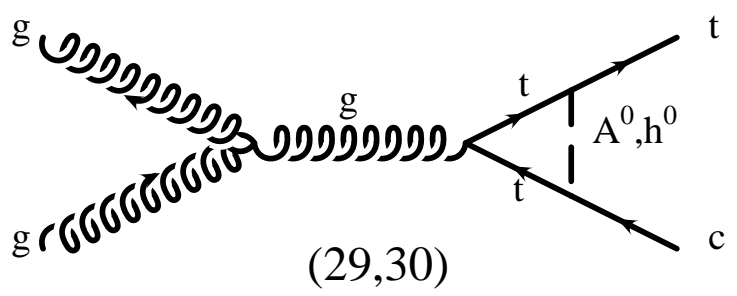

Fig.1 (a) 


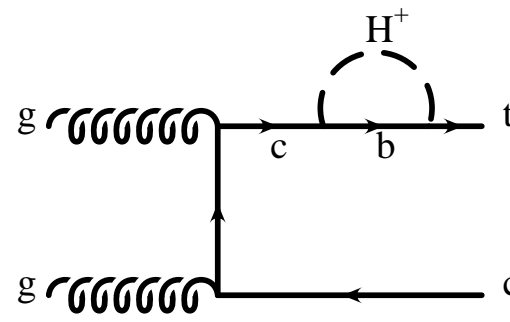

c (1)

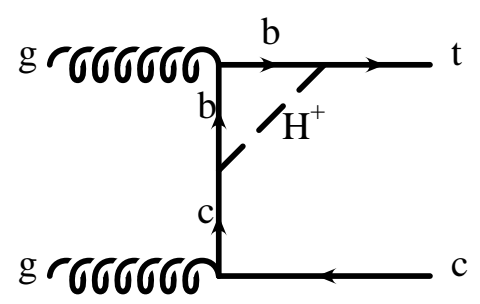

$(7,8)$

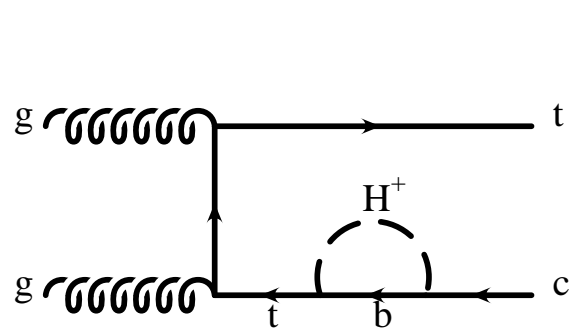

$(3,4)$

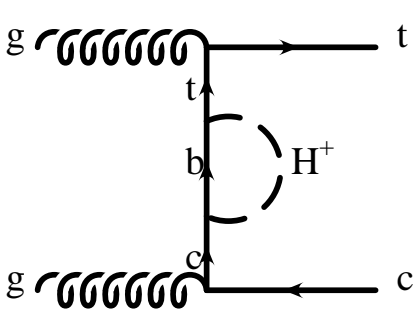

$(5,6)$

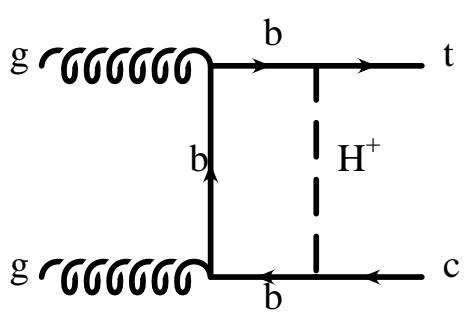

$(11,12)$
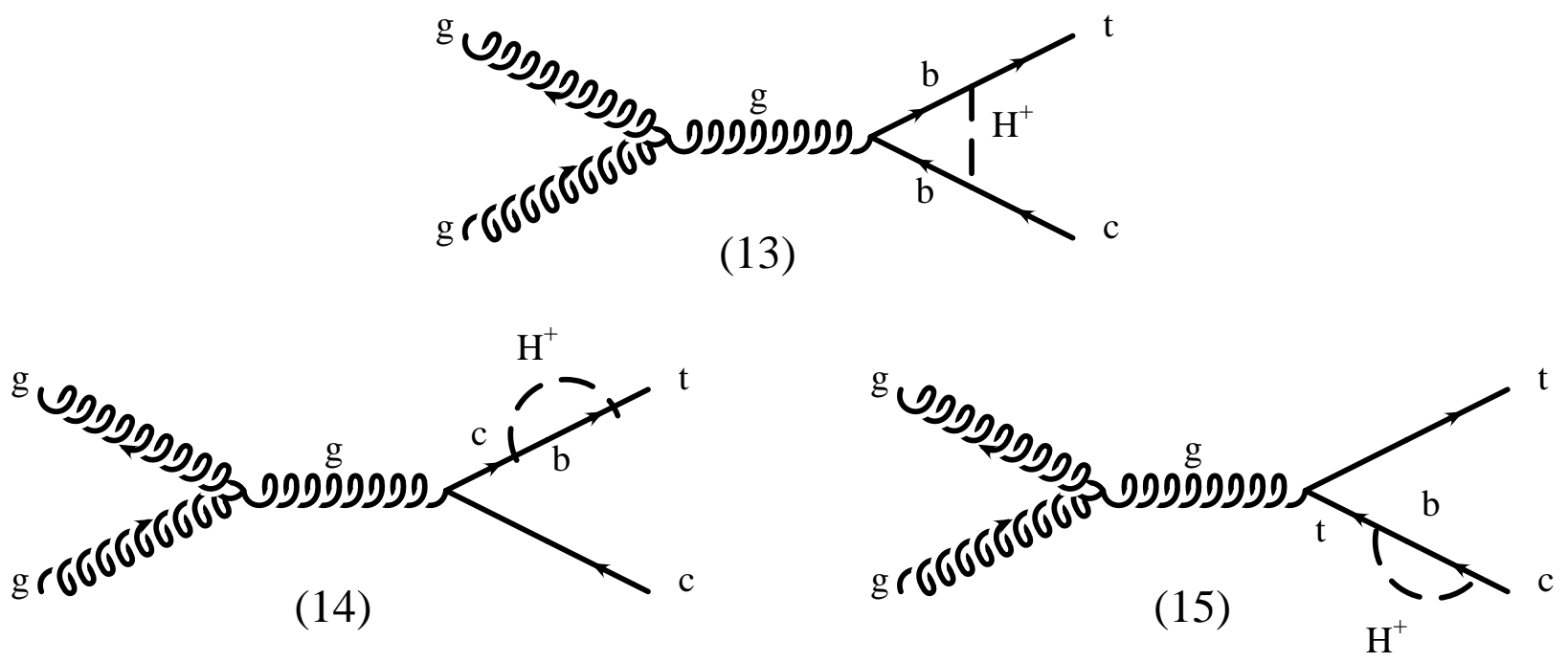

Fig.1 (b) 
Fig.(2)

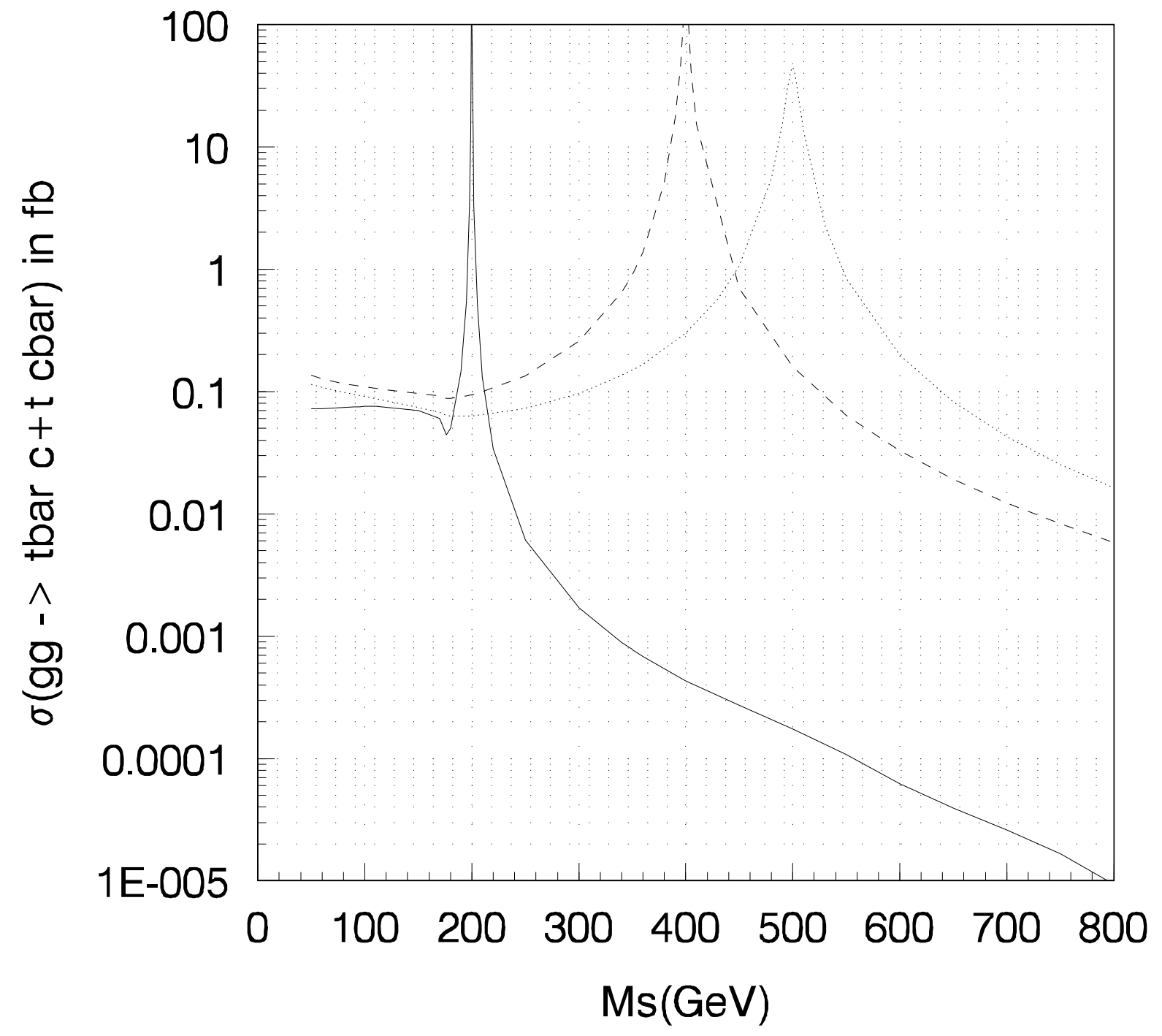


Fig.(3)

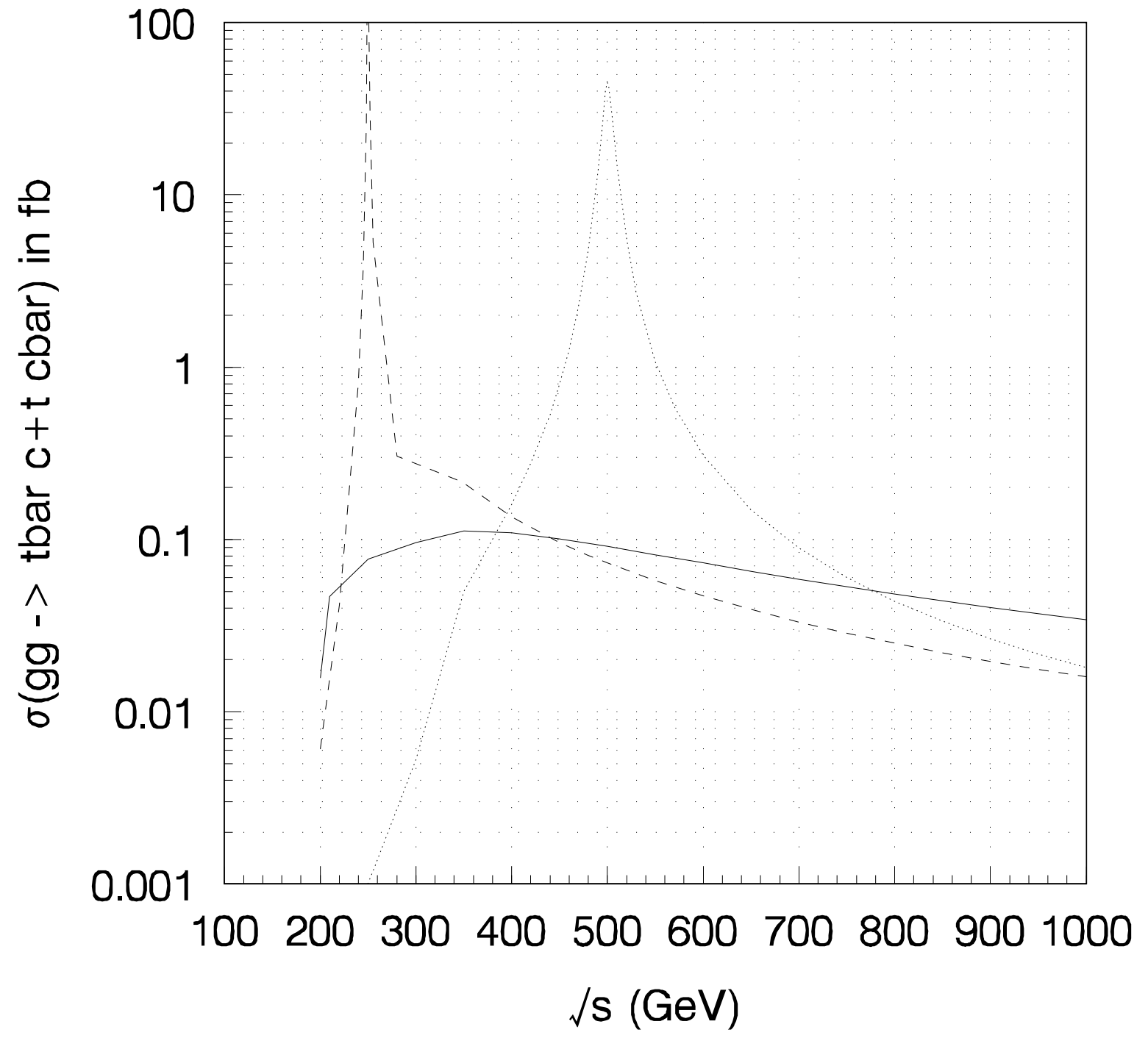


Fig.(4)

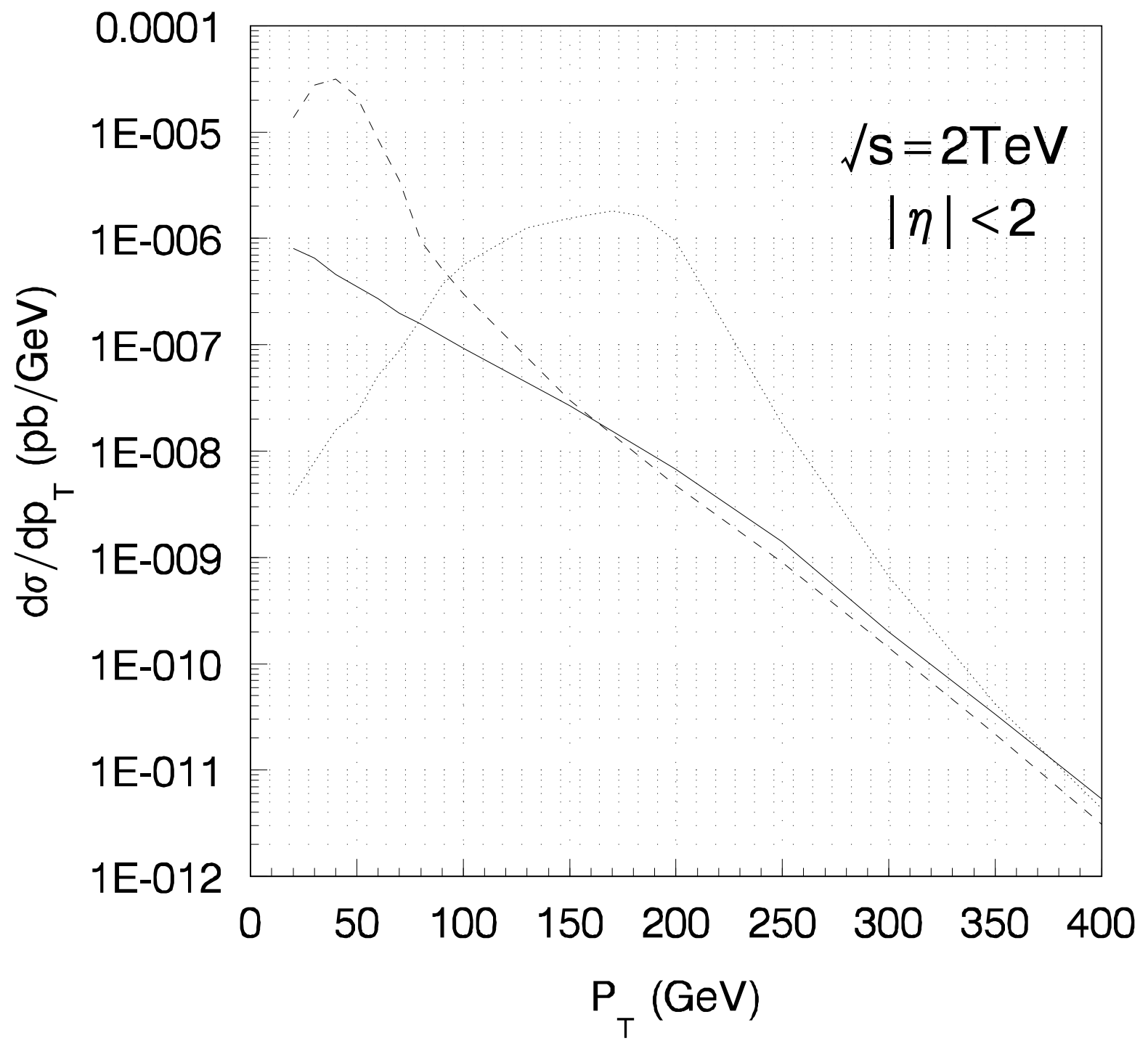


Fig.(5)

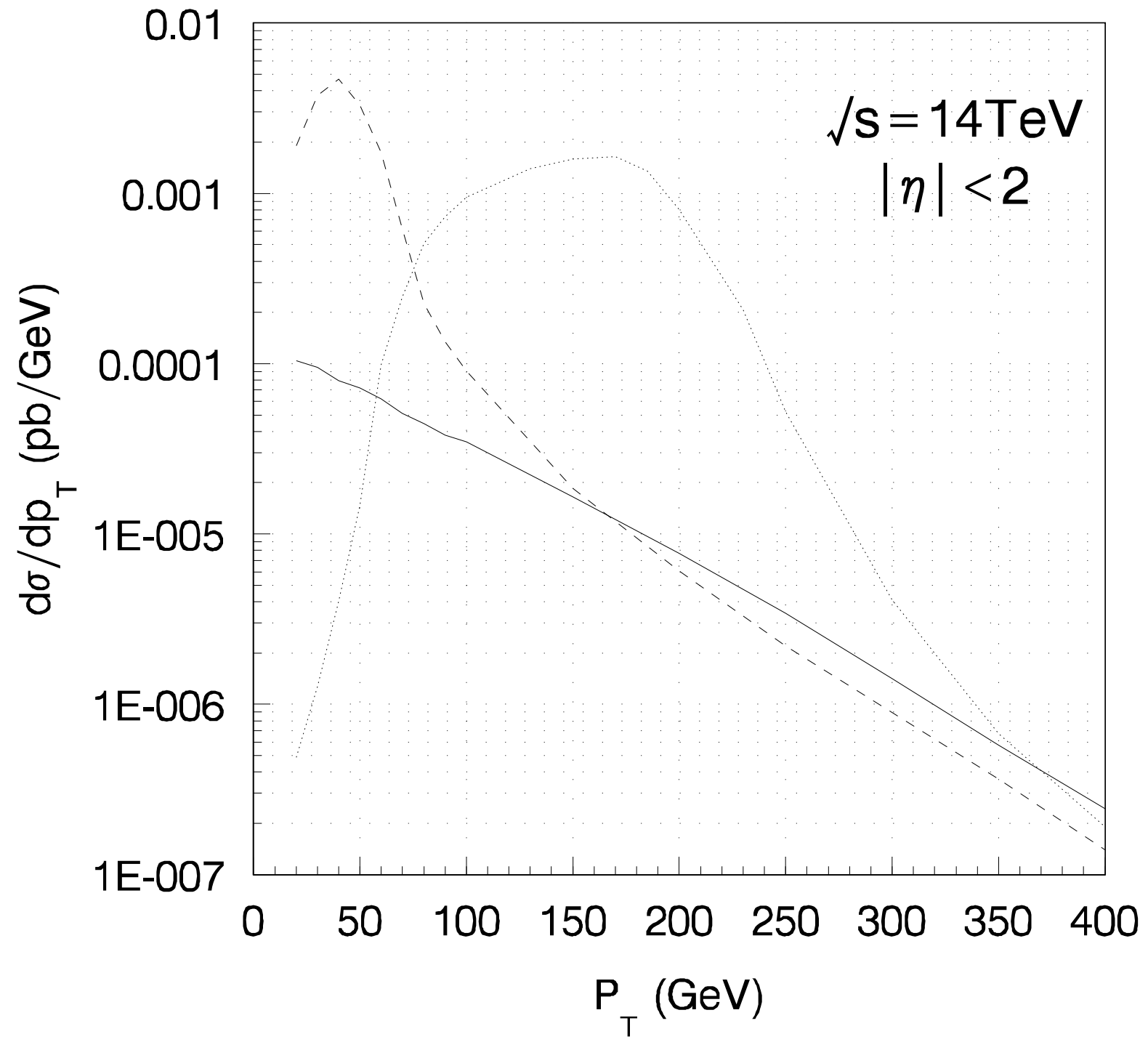


Fig.(6)

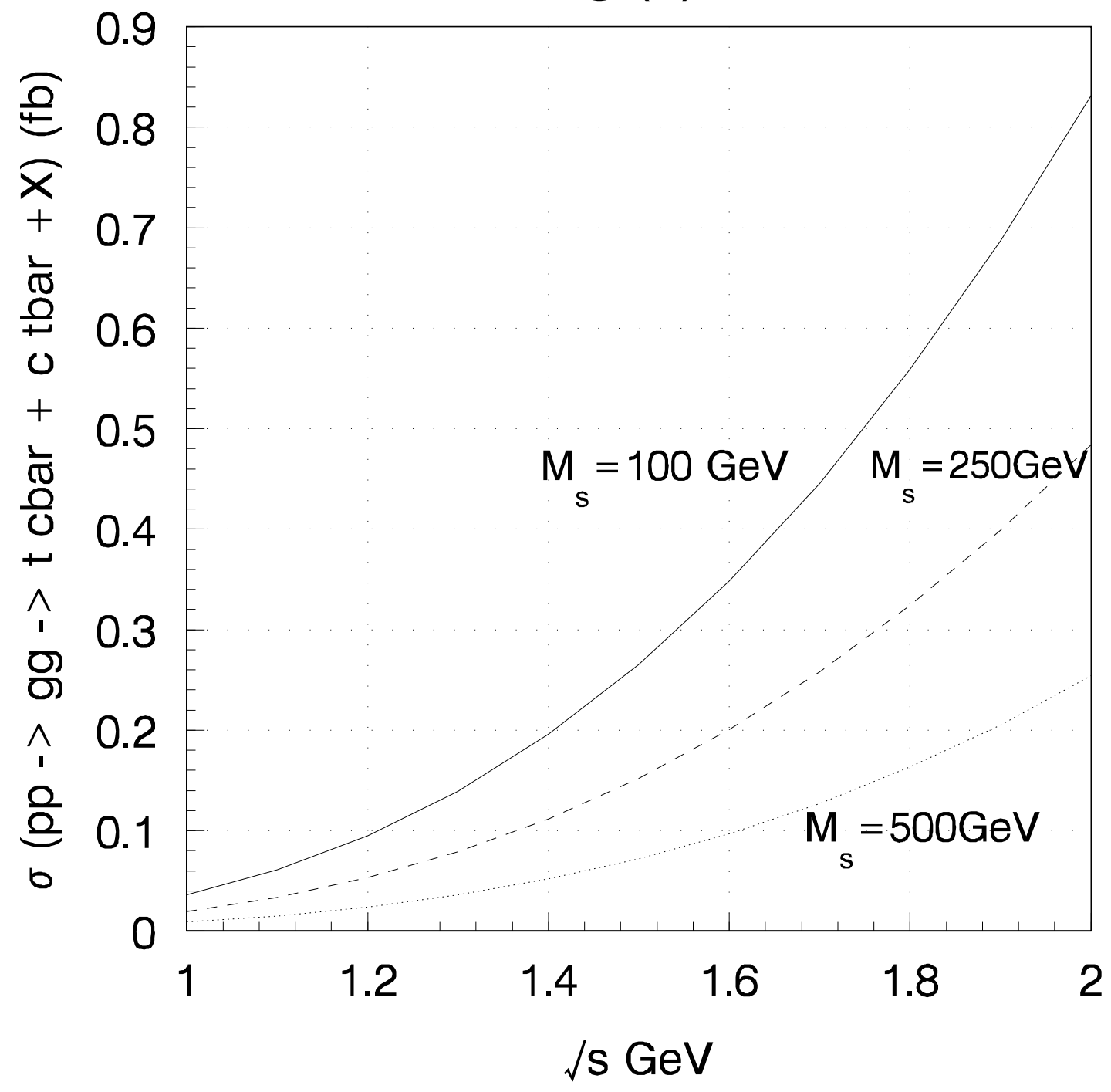


Fig.(7)

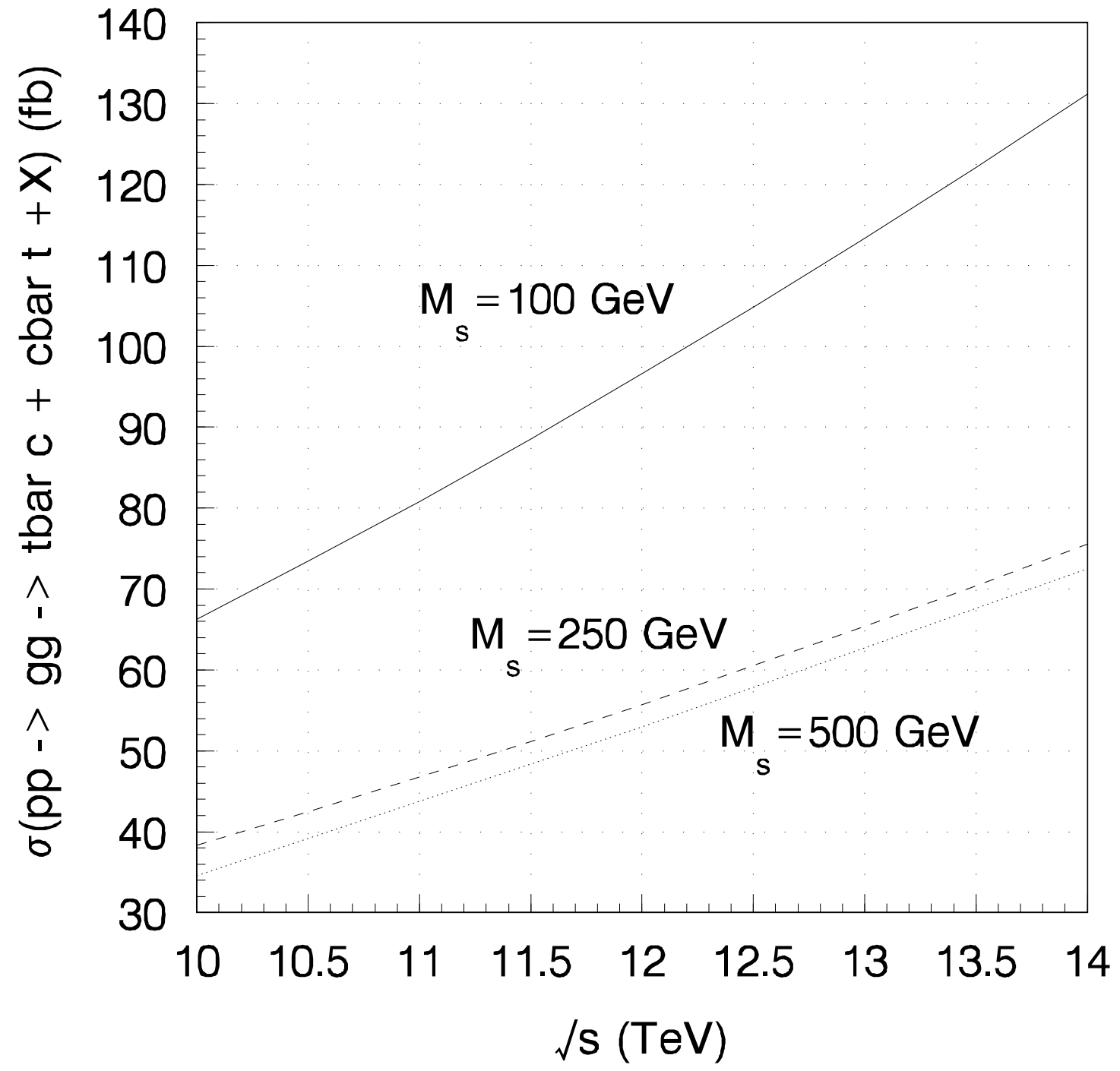

